\title{
FINGER-VEIN BASED BIOMETRIC SECURITY SYSTEM
}

\author{
Jose Anand ${ }^{1}$, T. G. Arul Flora ${ }^{2}$, Anu Susan Philip ${ }^{3}$ \\ ${ }^{1}$ Associate Professor, ${ }^{2,3}$ Assistant Professor, Department of Electronics and Communication Engineering, \\ KCG College of Technology, Chennai, India
}

\begin{abstract}
Finger vein recognition is a kind of biometric authentication system. This is one among many forms of biometrics used to recognize the individuals and to verify their identity. This paper presents a finger vein authentication system using template matching. Implementation using Matlab shows that the finger vein authentication system performs well for user identification.
\end{abstract}

Keywords - biometric, feature extraction, figure vein, security system.

\section{INTRODUCTION}

Finger vein [13] biometric authentication is a recent identification system in this modern era. This technology is used for wide variety of applications including credit card authentication, automobile security, employee time and attendance tracking, computer and network authentication, and so on.

Like fingerprints [12] or iris patterns, finger vein based blood vessel patterns are unique for each individual. Finger vein based blood vessel pattern have high security because the veins are located under the surface of the skin. The fingerprints can be cheated by dummy finger fitted with a copied fingerprint, but the finger vein based identification system is highly secure for authentication.

The iris pattern recognition [5] is known for low error rates of authentication, but some users feel psychological resistance to the direct application of light rays into their eyes. In addition to this, precise positioning of the eyes is required for accurate iris authentication. So the iris authentication system is provided with high-cost position adjustment mechanisms for the accurate recognition.

For authentication application the pattern of the finger vein [14] is stored in a database. The finger is placed on an attester terminal which contains a near-infrared, light emitting diode light source and a monochrome charge coupled device camera. The hemoglobin present in the blood absorbs the near infrared light emitting diode light and makes the vein to appear as dark pattern. The recorded image is digitized and stored in the database. During authentication, the finger vein is scanned and is compared with the image in the database.

The rest of the paper is organized as follows. Section 2 reviews about the related literature and section 3 describe the finger-vein based security system for authentication needed real-time applications. Section 4 details the performance evaluation of finger-vein security system using Matlab tool, and finally conclusion and future scope is given in section 5 .

\section{RELATED WORK}

In this section, we review the prior work on finger vein biometric security system over various applications. David et al [1] introduced preliminary process to enhance the image quality that worsen by light effect and produces noise by the web camera, then segmented the vein pattern by using adaptive threshold method and matched them using improved template matching. The result shows that even the image quality is not good and as long as the veins are clear with some appropriate process can be used for personal identification.

Wenming et al [2] proposed a structured personal identification approach using finger vein Location and Direction Coding (LDC). Initially finger vein imaging device is designed using Near-InfraRed (NIR) light source, by which a database for finger vein images is established. The brightness difference in the finger vein image is used to extract the vein pattern. Then finger vein LDC creates a structured feature image for each finger vein. The structured feature image is utilized to conduct the personal identification with image database for finger vein, which includes 440 vein images from 220 different fingers.

Hua-Bin et al [3] presented an algorithm based on adaptive filtering and retinex method for enhancement of hand vein images. The principal of the near-infrared hand vein image acquisition is introduced, then the retinex method is used to normalize hand vein images, and the adaptive smoothing method is selected to estimate the illumination. Then the gray cosine transform is used to enhance the discrimination of the skin and the vein in hand vein images. Then a determination criterion of hand vein is established to remove the false vein blocks from the segmented hand vein images. 
Shi et al [4] proposed a method to make low cost devices using vein pattern images with low contrast, and high-quality images. The method could extract the vein network successfully as using high-quality images. The principle of vein imaging is discussed to acquire the vein images which could enhance the contrast and the algorithm of extracting the vein pattern from low quality images.

Desong et al [5] presented a more secure and reliable user identification mechanism using biometrics technology equipped into the consumer electronics devices. The system uses finger-vein identification system which provides high security and reliability than other identification technology. The algorithm composes of a feature extraction using radon transform and singular value decomposition and classification using a normalized distance measure.

Zhi et al [6] proposed a real-time embedded finger-vein recognition system for authentication on mobile devices. The system is implemented on a DSP platform and equipped with a novel finger-vein recognition algorithm. The system takes about 0.8 seconds to verify one input finger-vein sample and achieves an equal error rate of 0.07 percent on a database of 100 subjects. The results proved that the finger-vein recognition system is qualified for authentication on mobile devices.

Li et al [7] proposed a modality-based bi-finger vein verification system. Both the finger vein and finger shape could be extracted from the single image acquired from the sensor. The system includes the new finger vein network extraction algorithm. The intersection of the forefinger and middle finger as the origin is introduced to the coordinate system, proposing a method including determining the region of interest, the finger vein and shape features extraction and its corresponding fusion verification.

Lin et al [8] presented an algorithm for segmenting the dorsal hand vein image and extracting the vein skeleton. After gray and size normalizing, Gaussian low pass filter and median filter are used to eliminate the speck noise and the horizontal strip scanning noise respectively. Then an improved NiBlack algorithm segments the vein pattern and an area thresholding algorithm removes the noise blocks from the vein pattern.

Jinfeng et al [9] focused on finger-vein enhancement and segmentation based on Gabor filters in the spatial domain. Considering the high randomicity of the finger-vein networks, a bank of even symmetric Gabor filters with eight orientations is firstly used to exploit vein information in images. Then, image reconstruction is implemented to generate an image containing an integrated finger-vein network.

Gongping et al [10] proposed a finger vein recognition method based on a Personalized Best Bit Map (PBBM). The method is rooted in a local binary pattern based method and then inclined to use the best bits only for matching. The recognition framework consists of preprocessing, feature extraction, and matching. For evaluating the effectiveness of the method extensive experimental designs are made and results show that PBBM achieves better performance.

Naoto et al [11] proposed a method of personal identification based on finger vein patterns. An image of a finger captured under infrared light contains not only the vein pattern but also irregular shading produced by the various thicknesses of the finger bones and muscles. The method extracts the finger vein pattern from the unclear image by using line tracking that starts from various positions.

\section{FINGER-VEIN BASED SECURITY SYSTEM}

Finger vein structure is not easily seen in visible light. So the device to capture the finger vein image composed of Near InfraRed (NIR) Light Emitting Diodes (LED) of 850 nanometer wavelength and a Charge Coupled Device (CCD) camera [13], [14]. Figure 1 shows the flow diagram of the finger-vein based biometric security system.

The important step in finger-vein recognition is the vein extraction from the background. The finger-vein images are acquired by the use of NIR spectroscopy. The finger-vein image obtained from the NIR spectroscopy appears to be darker than the other regions of the finger. This is because the blood vessels alone will absorb the rays.

The performance of the finger-vein extraction and matching algorithm depends upon the quality of the input image. Initially the image is enhanced to eliminate the noise using oriented filter method. This also enhances the ridgelines, and uses Gabor filter. Gabor filters are band-pass filters that are having both orientation selective and frequency selective properties. These utilize the directionality feature of the finger-vein image and then the finger-vein is extracted from the enhanced oriented filter image.

The feature vector is obtained by taking mean, standard deviation and co-occurrence parameters. Let $\operatorname{Ri} \theta(\mathrm{x}, \mathrm{y})$ be the component image corresponding to $\theta$ or sector $\mathrm{Si}$. For $\mathrm{i}=0$, $1,2 \ldots 47$ and $\theta \in[0 \mathrm{o}, 45 \mathrm{o}, 90 \mathrm{o}, 135 \mathrm{o}]$. The features Mean $(\mathrm{Mi} \theta)$ and Standard deviation $(\mathrm{Fi} \theta)$, can be defined using equations (1) and (2) respectively.

$$
\begin{aligned}
& M i \theta=\frac{1}{k} \sum_{i=1}^{k} S i \\
& F i \theta=\sqrt{\left(\sum_{k} R_{i \theta}(x, y)-M i \theta\right)}
\end{aligned}
$$


The co-occurrence matrix $(\mathrm{C})$ is given by,

$$
C(i, j)=|\{((r, s),(t, v)) ; I(r, s)=i, I(t, v)=j\}|
$$

Where, $\mathrm{k}$ is the number of pixels in $\mathrm{Si}, \operatorname{Ri} \theta$ is the sector of the filtered image, and $M i \theta$ is the mean of that sector.

The grey level value in each sector of the filtered image is given as the finger code. Also the co-occurrence matrix (C) with distance $(1,1)$ i.e., one pixel below and one pixel right, in each sector a feature, and contrast is calculated using the cooccurrence matrix as given in equations (3) to improve the recognition rate of this authentication system.

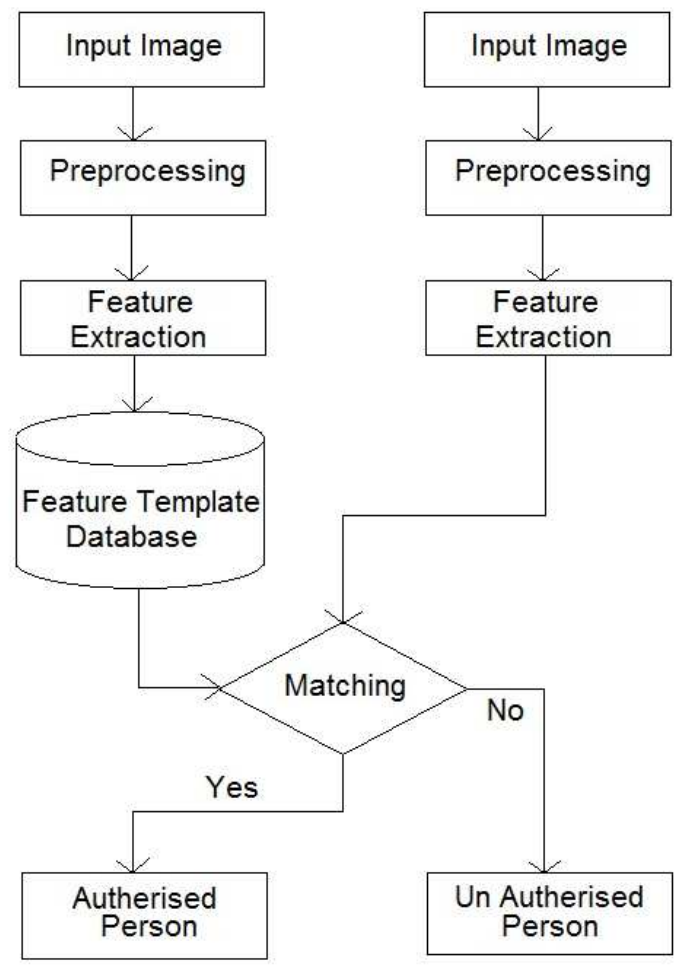

Fig 1 Flow diagram of finger-vein security system

\section{PERFORMANCE EVALUATION}

Analysis of finger-vein based biometric security system has been carried on an Intel Core 2 Duo CPU system with 2.10 $\mathrm{GHz}$ on a 32-bit Windows 7 Ultimate Operating System using MATLAB. Since there is no finger-vein image database is available, a database for 50 people between 21 years and 55 years old. From each people the forefinger, middle finger, and ring finger of both hands are considered. The image which is obtained from the real-time camera is shown in figure 2 . The image after preprocessing is shown in figure 3 .

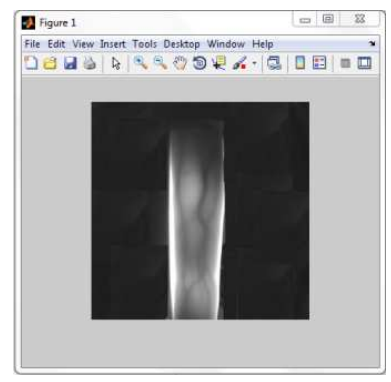

Fig 2 Input Image

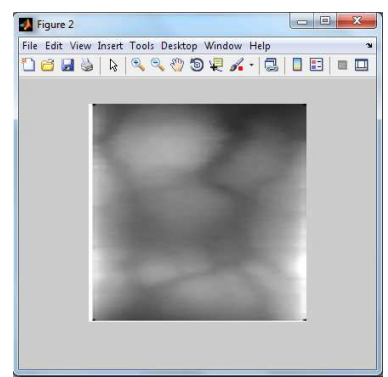

Fig 3 Preprocessing

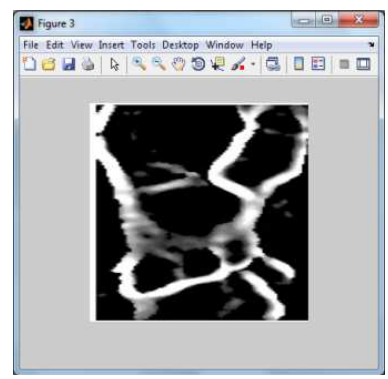

Fig 4 Feature Extraction

While matching two types of errors results in the finger-vein based biometric verification security system. The errors are false rejection rate and the false acceptance rate. False rejection is a claim that a genuine image is considered as impostor. False acceptance is a claim that an impostor image is considered as genuine. When the false rejection rate and the false acceptance rate are equal, then the performance of the system is evaluated as equal error rate. This system is suitable for mobile device applications with low computational complexity and low power consumption.

\section{CONCLUSIONS}

In this paper, we presented a finger-vein based biometric security system that can be used for security based electronic devices. The method can extract the finger-vein feature for recognition from the NIR images. This method uses single sample and is convenient to the application. This work can be extended with increasing the database for further verification. 


\section{REFERENCES}

[1] David Mulyono, and Horng Shi Jinn, "A Study of Finger Vein Biometric for Personal Identification", Proceedings of the IEEE International Symposium on Biometrics and Security Technologies (ISBAST 2008), pp. 1-8, 2008.

[2] Wenming Yang, Qing Rao, and Qingmin Liao, "Personal Identification for Single Sample using Finger Vein Location and Direction Coding", Proceedings of the IEEE International Conference on Hand-based Biometrics (ICHB), pp. 1-6, 17-18 March 2011.

[3] Hua-Bin Wang, and Liang Tao, "Novel Algorithm for Enhancement of Hand Vein Images based on Adaptive Filtering and Retinex Method", Proceedings of the IEEE International Conference on Information Science and Technology (ICIST), Wuhan, Hubei, China, pp. 857-860, 23-25 March 2012.

[4] Shi Zhao, Yiding Wang, and Yunhong Wang, "Extracting Hand Vein Patterns from Low-Quality Images: A New Biometric Technique using Low-Cost Devices", Fourth International Conference on Image and Graphics (ICIG 2007), IEEE Computer Society, pp. 667-671, 22-24 Aug 2007.

[5] Desong Wang, Jianping Li, and Gokhan Memik, "User Identification based on Finger-vein Patterns for Consumer Electronics Devices", IEEE Transactions on Consumer Electronics, Vol. 56, No. 2, pp. 799-804, May 2010.

[6] Zhi Liu, and Shangling Song, "An Embedded RealTime Finger-Vein Recognition System for Mobile Devices", IEEE Transactions on Consumer Electronics, Vol. 58, No. 2, pp. 522-527, May 2012.

[7] Li Zhichao, Sun Dongmei, Liu Di, and Liu Hao, "Two Modality-Based Bi-Finger Vein Verification System", 2010 IEEE $10^{\text {th }}$ International Conference on Signal Processing (ICSP) pp. 1690-1693, 24-28 Oct 2010.

[8] Lin Yang, Xiangbin Liu, and Zhicheng Liu, "A Skeleton Extracting Algorithm for Dorsal Hand Vein Pattern", 2010 International Conference on Computer Application and System Modeling (ICCASM 2010), pp. V13-92-V13-95, 22-24 Oct 2010.

[9] Jinfeng Yang, Jinli Yang, and Yihua Shi, "Finger-Vein Segmentation Based on Multi-channel Even-symmetric Gabor Filters", IEEE International Conference on Intelligent Computing and Intelligent Systems 2009 (ICIS 2009), Vol. 4, pp. 500-503, 20-22 Nov 2009.

[10] Gongping Yang, Xiaoming Xi, and Yilong Yin, "Finger Vein Recognition Based on a Personalized Best Bit Map”, Journal on Sensors, Vol. 12, pp. 1738-1757, doi: 10.3390/s120201738, 2012.

[11] Naoto Miura, Akio Nagasaka, and Takafumi Miyatake, "Feature Extraction of Finger-vein Patterns based on Repeated Line Tracking and its Application to Personal Identification", Machine Vision and Applications, Vol. 15, pp. 194-203, 2004.
[12] Lee H, S. H. Lee, T. Kim, and H. Bahn, "Secure User Identification for Consumer Electronics Devices", IEEE Transactions on Consumer Electronics, Vol. 54, No. 4, pp. 1798-1802, Nov. 2008.

[13] Hashimoto J., "Finger Vein Authentication Technology and its Future", 2006 Symposium on VLSI Circuits, Digest of Technical Papers, pp. 5-8, 2006.

[14] Wu J. D., and S. H. Ye, "Driver Identification using Finger-Vein Patterns with Random Transform and Neural Network", Expert System Applications, Vol. 36, pp. 5793-5799, 2009. 